THE

\title{
MEDICO.PSYCHOLOGICAL ASSOCIATION OF GREAT BRITAIN AND IRELAND.
}

\author{
THE COUNCIL, I898-9. \\ PRESIDENT.-A. R. URQUHART, M.D. \\ PRESIDENT ELECT.-J. B. SPENCE, M.D. \\ EX-PRESIDENT. -T. W. MCDOWALL, M.D. \\ EMERITUS TREASURER. - JOHN H. PAUL, M.D. \\ TREASURER. - H. HAYES NEWINGTON, F.R.C.P.Ed.
}

EDITORS OF JOURNAL.

\begin{tabular}{l|l} 
HENRY RAYNER, M.D. & CONOLLY NORMAN, F.R.C.P.I. \\
A. R. URQUHART, M.D. & EDWIN GOODALL, M.D.
\end{tabular}

AUDITORS. $\left\{\begin{array}{l}\text { T. SEYMOUR TUKE, M.B. } \\ \text { T. OUTTERSON WOOD, M.D. }\end{array}\right.$

Divisional SECRETARY FOR SCOTLAND.-A. R. TURNBULL, M.B.

DIVISIONAL SECRETARY FOR IRELAND.-A. D. O'C. FINEGAN, L.R.C.P.I.

DIVISIONAL SECRETARY FOR NORTHERN AND MIDLAND DIVISION.-W. CROCHLEY CLAPHAM, M.D.

DiVisionAl SECRETARY FOR SOUTH-EASTERN DIVISION. - E. W. WHITE, M.B.

DIVISIONAL SECRETARY FOR SOUTH-WESTERN DIVISION.-P.W. MACDONALD, M.D.

GENERAL SECRETARY.-ROBERT JONES, M.D., B.S., F.R.C.S.

SECRETARY OF EDUCATIONAL COMMITTEE.-C. A. MERCIER, M.B. REgISTRAR.-J. B. SPENCE, M.D.

MEMBERS OF COUNCIL.

FLETCHER BEACH, M.B. 1896. WILLIAM R. WATSON, L.R.C.S.1897

R. PERCY SMITH $M$.

D. M. CASSIDY, M.D.

R. D. HOTCHKISS, M.B.

H. A. BENHAM, M.D.

$"$ ALFRED TURNER, M.D.

E. T. EWART, M.D.

A. LAW WADE, M.D.

A. W. CAMPBELL, M.D.

JAMES M. MOODY, M.R.C.S. 1897. T. S. SHELDON, M.B.

S. R. MACPHAIL, M.D.

J. G. SOUTAR, M.B.

JAMES CHAMBERS, M.D.

", OSCAR T. WOODS, M.B.

EXAMINERS.

ENGLAND $\{$ C. A. MERCIER, M.B.

$\{$ R. PERCY SMITH, M.D.

SCOTLAND $\{$ J. RORIE, M.D.

IRELAND $\left\{\begin{array}{l}\text { OSCAR T. WOODS, M.D. } \\ \text { CONOLLY NORMAN, F.R.C.P.I. }\end{array}\right.$

PARLIAMENTARY COMMITTEE.

DR. FLETCHER BEACH
DR. BENHAM.
DR. G. F. BLANDFORD.
DR. D. M. CASSIDY.
DR. CLOUSTON.
DR. E. M. COOKE.
DR. GARDINER HILL.
DR. ROBERT JONES.
MR. LEY.
DR. J. G. MCDOWALL.
DR. MERCIER.

DR. H. HAYES NEWINGTON.

DR. YELLOWLEES

With power to add to their number.

XLV.

DR. CONOLLY NORMAN.

DR. EVAN POWELL.

DR. RAYNER.

DR. SAVAGE.

DR. PERCY SMITH.

DR. J. B. SPENCE.

DR. STOCKER

DR. G. THOMSON.

DR. URQUHART.

DR. WHITCOMBE.

DR. WHITE.

DR. WIGLESWORTH. 


\title{
INDEX MEDICO-PSYCHOLOGICUS.
}

\section{3-4.}

\author{
Preparte by Dr. J. 'Turner.
}

Absinthe. Beitrag zur Lehre von der nbsteigenden Degeneration in Gehirn und Rückenmark und Bemerkungen über die Localisstion und die Leitungs. bahnen der Krämpe bei der Absynth-Epilepsie. R. Boyce. Neurol. Centralbl., Leipz., 1894, xiii, 466-470.

Address. Some remarks on the address delivered to the American Poychological Aecociation, by 8. Weir Mitchell, M.D., May 16th, 1894. Am. J. Insan., Chicago, 1894-5, li, 171-181. W. Channing.

Addrew before the fiftieth Annual Meeting of the American MedicoPsychological Association, held in Philadelphia. S. W. Mitchell. May 16th, 1894. J. Nerv. and Ment. Dis., N.Y., 1894, xxi, 418-437.

Ages. Ages and death-rates of lunatics in the district where accuinulation of lunatics is most advanced. T. A. Chapman. J. Ment. Sc., Lond., 1894, xl, 485-393, $4 \mathrm{ch}$.

Alt Scherbitz. The great Asylum of Alt Scherbitz, near Leipzig. C. Norman. Ibid., 494.

Alienints. New England alienists of the last half-century. T. W. Fisher. Am. J. Insan., Chicago, 1894-5, li, 161-170.

Alimentary. Concerning some of the alimentary affections in the insane. Amelia Gilmore. Philnd. Hosp. Rep., 1893, ii, 198-205.

Aphonia. Preudo-hysterical aphonia. F. B. Eaton. J. Am. M. Ass., Chicngo, 1894, xxiii, 452.

Arteries. Sulla disposizione delle arterie della base dell' encefalo nei normali enegli alienati. A. Mori. Monitore zool. ital., Firenze, 1893, iv, 179-202.

Aeciai. S. François d'Aseise. Etude sociale et médicale. A. Bournet. Lyon, 1893, A. Storch, 198 pp., 2 pl., post 8vo.

Aoylums. Zur inneren Organisation der Irrenanstalten. Für Irrenărzte und hobere Verwaltungabeamte. Schnefer. Hamburg, 1894, O. Meienener, 19 pp. $12 \mathrm{mo}$.

Kjobenharns Sindssygeanstalt $\mathrm{i}$ forrige Aarkundrede. $\left[.,{ }^{\circ}\right.$. insane asylums in the last century.] Hosp.-Tid., Kjöbenh., 1893, 4 R., i. G. Norrie. 790-794.

Difficulties which prevent the realisation of Dr. Mitchell's ideal hospital for the insane. L. S. Hinckley. J. Nerv. and Ment. Dis., N.Y., 1894, xxi, 697-601.

Development of the present hospital for the insane. W. W. Godding. $\Delta \mathrm{m}$. J. Insan., Chicago, 1894, li, 64-70.

Insane asylum management. S. A. Cunningham. Cincinn. LancetClinic, 1894, n.s., Ixxiii, 520-522.

Amentia. [Case of periodical amentia.] A. J. Yuschenko. Arch. paichiat. \&c., Charkov, 1894, xxiii, 83-97.

$\Delta$ uto-intoxication. Des auto-intorications dans les maladies mentales. J. 86́glas. Arch. gén. de mód., Par., 1893, ii, 532-555.

Des anto-intorications dans les maladies mentales. Lavaure-Chevalier et Regis. [Rup.] Ann. de paychiat. et d'hypnol., Par., 1893, n.8., iii, 261-274.

Om Autointoksikationspychiater. D. E. Jacobsou. Biblioth. f. Laeger, Kjöbenh., 1894, 7 R., v, 158-186.

Ueber Antointoxications-Psychosen. Allg. Ztschr. f. Psychiat., \&c., Berl., 1894, li, 379-406. 
Antomatism. Automatisme ambulatoire. Ann. méd.-psych. R. Semelaigne. Par., 1894, 7 8., xix, 71-87.

- Antomatisme dans la période d'anra des attaques; variations spontanées de la sensibilité et surtout du champ visuel, correspondant aux phénomènes d'automatisme. Séglas. Cong. anı. de méd. ment., C.-r., 1892, Blois, 1893, iii, 309-322.

Barbe Buvée. Barbe Buvée, en religion, scur de Sainte-Colombe, et la prétendue possession des Ursulines d'Auxonne (1658-1663); (6́tude historique et médicale, d'après des manuscrits de la Bibliothèque nationale et des Archives de l'ancienne province de Bourgogne). S. Garnier. Arch. de neurol., Par., 1894, xxvii, 352, 418; xxviii, 18, 111, 196, 296.

Blood. Ueber den Hänoglobingehalt und das specifische Gewicht des Blutes bei Geisteskranken. Vorster. Allg. Ztschr. f. Psychiat., \&c., Berl., 1893-4, $1,740.760$.

On the value of examination of the blood of the insane. J. A. Houston. Boston M. and S. J., 1894, cxxx, 53-56.

Body weight. Body weight and mental improvement. A. R. Moulton. Am. J. Insan., Chicago, 1894-5, li, 209-220.

Borderlands. Borderlands and crankdom. J. Weir, jun. Med. Rec., N.Y., 1894, xlv, 360-362.

Bunyan. The case of John Bunyan. J. Royce. Psychol. Rev., N.Y. and Lond., 1894, i, 22, 184, 230.

Care. Care of the insane in Finland. E. Hougberg. Am. J. Insanity, Utica, N.Y., 1893-4, i, 390-393.

Die năchste Aufgabe der Irrenpflege. Scholz. Allg. Ztschr. f. Psychiat., \&c., Berl., 1893-4, l, 690-704.

The care and trentment of the insane in private practice. J. T. Wilson. Virginia M. Month., Richmond, 1893-4, xx, 1100-1116.

Alienacäo mental após a extraç̧ão de cataracta dupla. Sou=a Refoios. Rev. de med. e cirurg., Lisb., 1894, i, 33.41.

Children. Causation and early treatment of mental disease in children. A. W. Wilmarth. J. Am. M. Ass., Chicago, 1894, xxiii, 271-274.

Climacteric. Climacteric insanity. G. L. Sinclair. Internat. Clin., Philad., 1894, 3 s., iv, 147-152.

The insanity of the climacteric period. E. Goodall. J. Ment. Sc., Lond., 1894, xl, 235-242.

Classification. Clasificación frenopática. Ots. Corresp. méd., Madrid, 1894, xxix, 155, 165, 172, 189, 196.

Colonies. Riu. C.-r., 1892, Blois, 1893, iii, 63-70, e G. Albertotti. Les colonies d'aliénés. Cong. ann. de mód. ment.

- [Colony of the insane at Lipova Mountain.] A. Ponomareff. Arch. psichiat., \&c., Charkov, 1894, xxiv, 67-91.

[Sur l'installation d'une colonie pour les aliénés au gouvernement Poltava.] A. Maltzeff, Zemsk. vrach, Poltava, 1893, vi, 584, 605, 625.

A New British epileptic colony. Brit. M. J., Lond., 1894, i, 1371.

Confusion. La confusion mentale. Henri Hannion. Par., 1894, G. Steinheil, 123 pp. 8vo.

De la confusion mentale primitive. J. Séglas. Arch. gén. de méd., Par., 1894, i, 538, 665.

Cowper. The life of William Cowper. [Rev.] T. Wright. J. Ment. Sc., Lond., 1894, xl, 79, 254.

Crank. The modern crank and mental responsibility. J. Punton. TriState M. J., Keokuk, 1893-4, i, 75-85.

Criminals. Osservazioni statistico-cliniche sui criminali-pazzi. G. Algeri. Arch. di psichist., \&c., Torino, 1894, xv, 408-411. Insanity among Criminals. H. E. Allison. Am. J. Insan., Chicago, 1894-5, li, 54-63.

- Die Unterbringung von geisteskranken Verbrechern. R. Schroeter. Allg. Ztschr. f. Psychiat., \&c., Berl., 1894, li, 432-446. 
Colonisation. Die Kolonisirung der Geisteskranken in Verbindung mit dem Ofien-Thür-System, ihre historische Entwickelung und die Art ihrer Ausführ. ung af Rittergut Alt-Scherbitz. Paetz (Albrecht), Berl., 1893, J. Springer, 252 pp., 6 pl., 1 plan, 8 vo.

Dante. Is nerrosi in Dante e Michelangelo. C. Lombroso. Arch. di psichiat., \&c., Torino, 1894, IV, 126.132.

Dante e la psichiatria : lettera à Cesara Lombroso. B. Chiara. Arch. di psichiat., \&c., Torino, 1894, xv, 455-462.

Degenerates. Un casd di teratofilia in un degenerato. Bull. d. sc. med. di Bologns. B. Galli-Valerio. 1893, 7 s., iv, 761-763.

- Dégénérescence. Traduit de l'allemand par Auguste Dietrich. 2 vols. Max Nordan. Par., 1894, F. Alcan, 437, 575 pp. 8 vo.

The need of apecial accommodation for the degenerate. J. Morel. J. Ment. Sc., Lond., 1894, xl, 597-605.

Dégénéreecence mentale et syndromes épisodiques multiples avec délire polymorpbe chez un même sujet. Magnan. Compt.-rend. Soc. de Biol., Par., 1894, 9 s., vi, pt. 2, 1-5.

- Les délires des dégénéres. Magnan. Bull. mó́d., Par.. 1894, viii, 73-76.

D6génórés et déséquilibrés. J. Dallemagne. Brux., 1894, H. Lamertin, $661 \mathrm{pp} .8 \mathrm{vo}$.

Delirium. Contributo all' etiologia e patogenesi del delirio acuto. Riforma med., Napoli, 1893, ix, pt. 3, 831-835.

Ueber den Wahn: eine klinische-psvchologische Untersuchung nebst einer Daratellung der normalen Intelligenvoorgänge. M. Friedmann. Wiesbaden, 1894, F. Bergmann, 207 pp. 8vo.

Stic nei (pazzi studiat in rapporto ul delirio. (Santangelo, F. Spoto.) Palermo, 1894, C. Clausen, 49 pp. 8 vo.

Dementia. Contribution à l'étude de la démence précoce. A. Vigouroux. Ann. mód.-psych., Par., 1894, 7 8., xix, 62-70.

- Irritative dementia, with' bouy thickening, following injury; temporary improvement. J. W. Springthorpe. Austral. M. J., Melbourne, 1893, n.s., xv.

- Démence progressive et inco-ordination des inouvements dans les quatre membres, chez deux enfante, le frère et la scur. Rev. neurol. (Boochaud), Par., 1894, ii, 2-7.

- Report of a case of syphilitic dementia. H. H. Herzog. North-west. Lancet, St. Paul, 1894, xiv, 167-169.

Dipeomania. De l'obsession et de l'impulsion en général, et de l'impulsion à boire en particulier (dipsomanie). Legrain. Ann. de la Policlin. de Par., 1894, iv, 201, 233.

Electricity. Sensibilita elettrica generale e dolorifica examinate col faradireometro in pazzi e normali. Arch. di psichiat., \&c. L. Roncoroni. Torino, 1893, xiv, 423-429, 1 pl.

Epileptics. Suite de la discussion des rapports de M. Masoin sur l'hospitalisation des épileptiques. Bull. Acad. roy. de Méd. de Bely., Brux., 1894, 4 8., viii, $154-182$.

Epilepsy. Epilepsy in a pnerpera with hyperpyrexia; death. T. Oliver. Lancet, Lond., 1894, i, 1295-1297.

- Ueber den Einfluss intercurrenter Krankheiten und physiologischer Processe auf die Epilepsie. E. Beckhaus. Ann. d. städt allg. Krankenh. zu München (1890-2), 1894, 187.218.

Psychical Epilepsy. S. J. Fort. Med. and Surg. Reporter, Philad., 1894, Ixx, 375.377.

L'occhio negli epilettici : studio anthropologico e inedico-legale. F. P. e G. Dotto. De Bono, Pisani, Palermo, 1893, xiv, 91.257, 1 pl.

- Ein Fall Von "Reflexepilepsie" beim Pferde. H. Därrwăchter Deutsche thierärztl. Wchnschr., Karlsruhe, 1894, ii, 17.

- The seat of origin and paths of conduction of the fits in absinthe epilepsy. R. Boyce. Brit. M. J., Lond., 1893, ii, 1097.

Il temperamento epilettico. F. Del Greco. Manicornio mod., Nocera, 1893, ix, 123-126. 
Ispilepay. Modiffcazioni della circolazione cerebrale nell' uomo nella epilessia e cotto l' azione di due nuovi furmaci ipnogeni; cloralamide e sulfato neutro di duboisina. V. Cupriati. Ann. di nevrol., Torino, 1893, n. 8., xi, 298.325, 1 ch. Epilepsy, Med. and Surg. Reporter, Philad. J. A. Larrabee. 1894, lxx, 447-450. Also, Am. Pract. and News, Lonisville, 1894, xvii, 208-214. Also, South M. Rec., Atlanta, 1894, xxiv, 175.182.

- Statistischer Beitra: zur Aetiologie der Aepilepsie. Liebe. Schweizerhof. - f. f. Nerv. u. Psych.-Kranke. weibl. Geschl., 4to, Berl., 1893, ii, 54-62. Die Epilepsia tarda. E. Mendel. Deutsche med. Wchnschr., Leıpz. u. Beri., 1893, xix, 1106-1108.

Tranquilli. Pensuto. Un caso di automatismo ambulatario postepilettico. Gazz. d. osp., Milano, 1893, xiv, 1122-1124.

- Sur un cas d'épilepsie Jacksonienne avec accès de tnchycurdie paroxistique de nature épileptoïde. A. Pitres. Arch. clin. de Bordeaux, 1894, iii, 97.112.

Ueber Epilepsie. Ztschr. f. d. Behandl. Schwachsinn. u. Epilept. Wildermuth. Dresd., 1894, n. F., x, 25, 41.

Epilepsie im Gefolge von Herzkrankheiten. H. Rosin. Med.-chir. Centralbl., Wien, 1893, xxviii, 608-610.

A revort of two cases of procursive epilepsy. C. S. Potts. Univ. M. Mag., Philad., 1893-4, vi, 696.

- Putient suffermg from Jacksonian epilepsy with sensory defect. A. Robertson. Glasgow M. J., 1894, xli, 450-453.

Zur Behandlung der Epilepsie nach Flechsig. L. Stembo. St. Petersb. med. Wchnschr., 1894, n. F., xi, 137.

Remarks on senile epilepsy. E. M. Sympson. Brit. M. J., Lond., 1894, i, 1069.

Dei caratteri poicopatici dell' epilessia. P. Saroli. Gior. med. d. r. esercito, \&c.. Roma, 1894, xliii, 532-537.

Combined motor and psychical epilepsy: report of a case. W. P. Spratling. Med. and Surg. Reporter. Philad., 1894, lxxi, 205-207. On epilepsy in early life, with especial reference to the colony system in the cure und treatment of epileptics. Med. News, Philad., 1894, 1xv, 291-295.

Fulie épileptique; ivresse pathologique. Delbastée. Presse méd. belye, Brux., 1894, xlvi, 257, 265.

Tipo compléto di pazzia morale a base epilettica. M. Carrara. Riforma med., Napoli, 1894, x, pr. 3, 520, 531.

Einige Bemerkungen über senile Epilepsie. E. M. Sympson. Internat. klin. Rnndschau, Wien, 1891, viii, 1013-1015.

Some remarks on epilepsy, and the cure of epileptics on the colony

plan. W. E. Drewry. Virginia M. Month, Richmond, 1894-5, xxi, 477-492. Notes on two hundred and fifty cases of epilepsy. R. K. Macalester.

N. York M. J., 1894, lix, 107, 237.

Neuere Arbeiten über Epilepsił. Seeliginüller. Deutsche med. Wchnschr., Leipz. u. Berl., 1894, xx, 12 ; 33, 62.

Zur Behandlung der Epilepsie. München med. Wchnschr., 1894, xli, 224, 251, 270. K. Alt.

L' asimmetria del cranio nell' epilessia del cavallo. R. Bassi. Arch. di psichiat., \&c., Torino, 1894, xv, 122.

Sobre las relaciones nosologicas entre el asma q la epilepsir. (Transl.)

M. G. Echeverria. Rev. de cien. meli., Habana, 1893, viii, 253-258.

Note sur les oscillations de poids chez les épileptiques. C. Féré. Compt.rend. Soc. de Biol., Par., 1893, 9 s., v, 891 . Note sur l'influence de l'érysipèle sur la marche de l'épilepsie. Ibid., 828-830.

Study of the caises, symptoms, nnd treatment of partial epilepsy. $R$. Macouzet. Alienist and Neurol, St. Louis, 1894, xv, 67.68.

Tremor saltatorius postepilepticur. W. Pascheles. Prag. med. Wchnschr., 1893, xviii, 593.

Epilepsy. F. E. Waxlıam. Denver M. Times, 1893-4, xiii, 311-317.

Ueber epilepsie, Wildermuth. Med. Cor.-Bl. d. württemb. ärztl. Ver., Stuttg., 1894, lxiv, 1-7.

The nuture of epilepsy. J. W. Anderson. Internat. Clin., Philad., 1893, 3 s., iii, 97-103. 
Epilepey. Remarks upon the medicinal treatment of chronic epilepsy. E. D. Bondurant. Am. J. Insan., Chicugo, 1894-5, li, 23.89.

- L'albuminuria post-epilettica. Gasz. med. lomb., Milano, 1894, xv, 181, 191, $201,211$.

I distarbi visivi degli epilettica. L. Cognetti. Gior. med. d. r. esercito, sc., Roma, 1894, xlii, 652-682.

Accessi epilettiformi per cicatrice deforme. Policlin., Roma, 1893-4, i, 258-268.

- Folie 6́pileptique ; ivresee pathologique. Delbast6e. Preseo mód. belge, Brux., 1894, xlvi, 257.

Caso di epilessia guarito colla belladonna e colla Sorrente elettrica. Riv. clin. e. terap., Napoli, 1894, xvi, 285.

- Note sur un poussin mort à la suite d'accès d'épilepsie. C. Féré. C.-rend. Soc. de Biol., Par., 1894, 10 s., i, 618.

Epilepsy dependent upon Phimosis. A. E. Garrett. Texas Cour.-Rec. Med., Dallas, 1893-4, xi, 295, 298.

The infantile causes of epilepsy. W. R. Gowers. Clin. J., Lond., 1894, iv, 809-312.

Reflexepilepsie nach Unfall. Knopf. Deutsche med. Wchnschr., Leips. u. Berl., 1894, xx, 681.

L' epilettico volgare e la responsabiltà. L. Lojacono. Rassegna clin. e statist. d. Villa di Salute di Palermo, 1893, iii, 79-93.

- Otto anni di epileseia d' origine riflessa ; guarigione coll' ablazione della

causa. L. Mazzoleni. Boll. d. Soc. med. prov. di Bergamo, 1894, v, 31.

- L'épilopaie tardive (epilepsia tarda). Mendel. en Ann. de psychiat. et d'hypnol., Par., 1894, n. e., iv, 236-238.

Treatment of epilepey by Solanum carolinense. W. J. Herdman. Tr. Mich. M. Soc., Detroit, 18y4, xviii, 198-212.

Ueber einen ungerohnlichen Fall von Epilepsia nocturna. F.

Iohmann. Allg. Ztechr. f. Paychiat., \&c., Berl., 1894, li, 370-378.

- Demonstration eines Falles von Rindenepilepsie, geheilt durch die

Trepanation. E. Leyden. Berl. klin. Wehnechr., 1894, xxxi, 839-841.

Eine Bemerkung aur Behandlung der Epilepsie. C. Moeli. Therap. Monatsh., Berl., 1894, viii, 436-442.

- The eye treatment of epilepsy. A. L. Ranney. N. York M. J., 1894, $1 \times, 407-409$.

- Ricerche analitiche sopra la presenza della creatinina nrlle orine degli epilettici. E. Rosai. Ann. di freniat., dcc., Torino, 1893-4, iv, 363-395.

L'occhio degli epilettici : studio antropologico e medico-legale. (F. P.) De Bono a G. Dotto. Arch. di ottal., Palermo, 1893.4, i, 129, 165, 197, 234, 277, $1 \mathrm{pl}$.

Sensory and peychical epilepsy. T. Diller. N. York M. J., 1894, lix, 898.

- Note sur un cas de sialorrbée épileptique. C. Féré. Compt.-rend. Soc. de Biol., Par., 1894, 10 s., i, 258.

Un caso di tremore epilettico. A. Giannelli. Riforma med., Napoli, 1894, $x$, pt. 1, 651-653.

- $A$ case of procursive epilepsy. A. W. Hoisholt. Occidental M. Times, Sacrainento, 1894, viii, 177-184.

- Nye undersögelser over epilepsi med. demonstrationer af experimenter paa dyr. H. Holm. Tidsekr. f. d. norske Laegfor., Kristiania and Kjøbenb., 1894, xiv, 1-6.

Epilepsie in Folge einer Herzkrankheit. M. Hubergritz. Internat. klin. Rundschan, Wien, 1894, viii, 271-274.

- Epileptiforme Anfalle, durch Veränderungen in den Nasenholen hervorgerufen : zwei Fälle. F. Kjelmall. Berl. klin. Wchnschr., 1894, xxxi, 816.

$\triangle \Delta$ Presidential Address on the influence of extreme slowness of palse in the causation of upileptiform collvulsions. J. S. Bristowe. Lancet, Lond., 1894, ii, 671-673.

- Gravi manifestazioni isteriche in un epilettico. L. De Martiis Cognetti. Gazz. d. osp., Milano, 1894, xv, 899. 
Epilepsy. Some recent measures in the treatment of epilepsy, with special reference to the use of opium: " year's experience with Flechsig's plan. J. Collins. Med. Rec., N. York, 1894, xlvi, 355-360.

- Nocturnal Epilepsy. F. W. D'Evelyn. Tr. M. Soc. Calif., San Franc., 1894, 218-222.

- De l'exhibition cliez les épileptiques. H. Pribat. Par., 1894, G. Steinheil, 60 pp. 8 vo.

Ueber die Behandlung der Epilepsie, insbesondere mit Opium-Brom nach Flechsig. Carl Salzburg. Leipz., 1894, Veit and Co., 60 pp. 8vo.

- Epilepsy, at times a bladder reflex. H. K. Aiken. Med. Rec., N. Y., 1894, xlvi, 556.

Ueber die Bedeutung der corticalen Epilepsie für die topische Diagnostic der Gehirnkrankheiten. St. Petersb. med. Wchnschr. K. Dehio, 1894, n. F., xi, 325-328.

La epilepsia nocturna y sus diferencias del sonambulismo. Rev. de cien. mód., Habana, 1894, ix, 233.242. M. G. Echeverria.

An address on the dynamics of life in relation to the nature of epilepsy. W. R. Gowers. Lancet, Lond., 1894, ii, 1015, 1080.

Note on convulsive seizures. W. H. Hattie. Maritime M. News, Halifax, 1894, vi, 389-393.

- Die staatliche Fürsorge für Epileptiker. G. W. Jackoby. N. Yorker med. Monatsschr., 1894, vi, 347-353.

- [Typical cases of cortical epilepsy.] A. Y. Kozhevnikoff. Med. Obzr., Mosk., 1894, xlii, 97.118.

- The treatment of epilepsy, with notes on the influence of Solanum carolinemse in controlling the seizures. Indiana M. J., Indianap., 1894-5, siii, 149-154.

Sulla genesi corticale della epilessis. P. Penta. [Abstr.] N. riv. di psichiat., \&c., Napoli, 1894, ii, 12-15.

Een geval van reflex-epilepsie. J. Rotgans. Nederl. Tijdschr.v. Geneesk., Amst., 1894, 2 R., xxx, pt. 2, 112-118.

- Ueber Kinder-Epilepsie und ihre chirurgische Behandlung. R. Wichmann. Aerztl. Prakt., Hamb., 1894, vii, 1033, 1057.

A remarkable case probably allied to epileptic automatisin. W. S. Colman. Lancet, Lond., 1894, ii, 128.

Automatisme comitial ambulatoire. Roskam. Ann. Soc. méd.-chir. de Liège, 1894, xxxiii, 275-282.

- An epileptic (?) fit folluwed by forty-six hours unconsciousness. F. Roth. Lancet, Lond., 1894, ii, 190.

Obscure forms of epilepsy. M. P. Sexton. Med. Rev., St. Louis, 1894, xxx, 66 .

- Distribution géographique de l'épilepsie. Gélineau. I. d'hyg., Par., 1894, xix, 301, 315, 327.

De l'épilepsie thalamique. W. A. Hammond. Ann. de psychiat. et d'hypnol., Par., 1894, n. 8., iv, 33, 68.

Epileptics. State case of epileptics. M. N. Voldeng. Tr. Iowa M. Soc., Omaha, 1893, xi, 35.

On the care of epileptics. Petersou. Am. J. Insan., Utica, N.Y., 1893-4, i, 362-371.

Erotomania. Curieuses érotomaines. Hospital. Ann. méd.-psych., Par., 1894, 7 s., xix, 55-61.

Feeble-minded. Feeble-minded children. Nineteenth Cent., Lond. Sir D. Galton. 1894, xxxv, 276.283.

Feeding. Artificial feeding of the insane. J. Neil. Brit. M. J., Lond., 1894, i, 883.

Artificial feeding in acute melancholia. J. Neil. Brit. M. J., Lond., 1894, i, 183.

The forcille feeding of the insane. W. W. Herbert. Brit. M. J., Lond., 1894, i, 462.

Folie à Deux. A propos de la folie à deux. F. L. Arnaud. France méd., Par., 1893, xl, 690-692. 
Folie a Deux. De la folie à deux et de ses trois grandes rariétés cliniques. E. Montyel de Marandon. Gaz. d. hôp., Pur., 1894, lxvii, 702, 724, 752, 762.

Gastric Juice. Sulla tossicizà del succo gastricn delgi alienati. E. Masetti. Riv. sper. di freniat., Reggio-Emilin, 1894, xx, pt. 2, 204-217.

Genins. Genius and degeneration. J. Weir, jun. Med. Repr., Lond., 1894, v, 118.

Les infirmités du génie. Rev. de méd. lég. Cabanès. Par., 1893-4, i, 83-88.

Generative Organs. Some abnormal conditions of the generative organs associated with melancholia or mania. W. G. Wylie. Med. Rec., N. Y., 1894, xlvi, 185.

General Paralysis. Contribution a l'anatomie pathologique de ln paralysie générale. Joffray. Cong. ann. de méd. ment., C.-r., 1892 ; Blois, 1893, iii, 240-250.

Zur Frage über die allgemeine progressive Paralyse der Irren und ihre Behandlung bei den Syphilitikern. J. G. Platonnow. Centralbl. f. Nervenh. u. Psychiat., Coblenz and Leipz., 1893, n. F., iv, 505-518.

Note sur un cas de paralysie générule vraie consécutive à une encéphalo. pathie saturnine. C. Vallon. Cong. aun. de méd. ment., C.-r., 1892 ; Blois, 1893, iii, 259.

- Aetiologisches und Symptomatologisches zur Lehre von der progressiven Paralyse der Frauen. A. Westphal. Charité-Ann., Berl., 1893, xviii, 719-731.

- Progressive paralysis in women : its etiology ; special course of disease. J. D. Jedanoff. Arch. psichiat., \&c., Charkoff, 1893, xxii, No. 2, 86-87.

Case of marked improvement in general paralysis, with remarks on the treatment. H. Robertson. Glasyow M. J., 1893, xl, 414-418.

- Dementia paralytica sine paralysi. J. Belkowsky. Centralbl. f. Nerveul.. u. P.ychiat., Colleız und Leipz., 1894, n. F., v, 169.176.

The relative importance of the minute historical features of the brain cortex in general paralysis. E. C. Carter. Brain, Lond., 1893, xvi, 893-404.

Beiträge zur Kenntniss der Aptiolngie der progressiven Parulyse, mit besonderer Berücksichtigung der Syphilis. E. Hongberg. Allg. Ztsch. $f$. Psychiat., \&c., Berl., 1893-4, 1, 546-629.

Paralysie générale; lésions et symptômes spinaux; formes spinales. Mercredi med., Klippel. Par., 1894, v, 85-87.

Influenza della sifilide sulla paralisi progressiva. Gior, internaz. d. sc. med., Napoli, 1893, n. 8., xv, 732-748. M. Pelli.

- Zur Aetiolugie und Symptomatologie der progressiven Paralyse, mit besonderer Berücksuchtigung des Truumas und der im jugendlichen Alter vorkommenden Fälle von Paralyse. H. Gudden. Arch. f. Psychiat., Berl.. 1894, xxvi, 430-471.

The trophoneuroses of paretic dementia. F. C. Hovt. Am. J. Insun., Chicago, 1894-5, 1i, 51-53.

Efetti di prolungate e ubbondunti suppuzazioni nella paralisi progressiva, con applicazioni terapeutiche. A. Marro e A. Ruata. Gior. d. R. Accad. di Med. di Torino, 1894, 3 s., xlii, 381-387.

De la forme infuntile de la paralysie générale. A. Moussons. Méd. inf., Par., 1894, i, 449-452.

Progressive Paralyse im Pubertätsalter, München. med. Wchnschr., 1894, xli, 617-619.

Das Ulnarik-Syinptom bei Geisteskranken insbesondere bei der progreasiven Paralyse. A. Cramer. München. med. Wchnschr., 1894, xli, $658,673$.

- Paralysie générnle à forme mélancolique d'emblée. Limousin méd., Limoges, 1894, xviii, 86.88.

Symptomes apasinodiques et coutractures permanentes dans la paralysie générale. Marc Frénel. Par., 1894, G. Steinheil, 135 pp. 8vo.

- Des lésions histologiques de la paralysie générale étudiées d'après lo méthode de Golgi. Azoulay et Klippel. Arch. de neurol., Par., 1894, xxviii, 81-91. 
General Paralysis. Intorno a un caso di demenza paralitica combinata con afasia. G. Mingazziui. Bull. d. K. Acad. Med. di Roma, 189:-3, xix, 156-167.

- Anutomie et physiologie pathologiques de la paralysie géuérale. Bull. méd., Par.. 1894, viii, 553-555. Joffroy.

Les hallucinations dans la paralysie générale. Jacques Barak. Par., 1894, G. Steinbeil, 101 pp. 8vo.

Dementia paralytica in the negro. H. J. Berkley. Johns Hopkins Hosp. Bull., Balt., 1893, iv, 94.97.

- Dementia paralytica in the negro race. H. J. Berkley. Jishns

Hopkins Hosp. Kep., Balt., 1894, iv, 171-208, 1 pl.

A case of general paralysis of the insane at the time of puberty. H. C. Bristowe. Brit. M. J., Lond.

On general paralysis (with a critical digest). W. J. Mickle. Brain, Lond., 1893, xvi, 50-85.

General paresis, a toxine disease? H. M. Ban:ister. Am. J. Insun., Utica, N. Y., 1893.4, 1, 477-492.

- Sequel of a case of general paralysis of the insane at puberty. H. C. Bristuwe. Brit. M. J., Lond., 1894, i, 1125.

Remarks upon twenty-eight cases of adult female geru-rul paralysis admithed to the Royal Edinburgh Asylum during the tive years 1889 to 1893. F. A. Elkins. Lancet, Lond., 1894, i, 1495-1497.

De la parulysie générale chez la femme; étiologie et clinique. J. D. Idanow. Anll. méd.-psych., Par., 1894, 7 s., xix, 382.457.

- Degenerative Muskelatrophio bei prouressiver Puralyse. Neurol. Centralbl., Leipz., 1894, xiii, 610-616. A. Hoche.

Ueber Zunahime und Ursachen der progressiven Paralyse. R. von Krafft-Eving. Internat. klin. Rundschan, Wien, 1894, viii, 1273-1279.

I. Réponse à l'observation de $M$. Henneguy relative au noircissement et à la conservation sous lumelles des coupes par les méthodes de Golgi a l'argent et au sublimé. II. Confirmation par la méthode de Cox des lésions cellulaires de l'écorre dans la paralysie générule. III. Aspect des cellules de Purkinje daus la parulgsie géuérale. Compt. rend. Suc. de Biol. L. Azonlny. Par., 1894, 10 8., i, 419.

- Les altérations des cellules de l'écorce cérébrale dans la paralysie générale, etudiées par la méthode de Golgi et Klippel. Azoulay. Ibid., 405-407.

- Paralysie générule; lésions et symptômes spinaux; formes spinales. Kippel. Arch. de méd. expér. et d'auat. puth., Par., 1894, vi, 75-114.

Arthropathy in general paresis. J. H. Lloyd. Phila. Hosp. Rep., 1893, ii, 164-175.

Etude comparative des lésions médullaires dans la paralysie générale et dans le tabes. P. Marie. Gaz. d. l,6̂p., Par., 1894, lxvii, 55-60.

- A contribution to the inorbid anatomy and pathology of the neuromuscular changes in genernl paralysis of the insane. A. W. Campbell. J. Ment. Sc., Lond., 1894, xl, 177-195, 2 pl.

- Des complications vikcérales de la paralysie générale progressive. G. Durante. Gaz. d. hôp., Par., 1894, lxvii, 213, 241.

- Des troubles trophiqueset des troubles circulatoires duns la paralysie générale. Grz. hebd. de inéd., Par., 1894, xli, 98, 111.

Goitre. Two cases of insanity with goitre treated with thyroid extract. T. S. McClsughry. J. Ment. Sc., Lond., 1894, xl, 635.

Hallucinations. Hallucinationerne. Biblioth. f. Laeger., Kjöbenh. K. Pontóppidan, 1893, 7 R., iv, 339.356.

p. ommmach $\mathrm{i}$ zudzeniach [Hallucinations and illusions]. W. Kohlberger. Przegl. lek., Krukow, 1893, xxxii, 517, 529, 546, 555, 581, 591, $605,618$.

Contributo allo studio delle allucinazioni verbali psico-motrici. Manicomio mod., Nocera, 1893, ix, 51-122. A. Piernccini.

Co-existences, associations et combinaisons hallucinatoires. J. Séglas.

J. d. conn. méd. prat., Par., 1894, 121, 131, 140.

The genesis of hallucinntion, illusion, and delusion. H. A. Tomlinson. J. Nerv. und Ment. Dis., N.Y., 1894, xxi, 576.585. 
Hallucinations. Hallucinations and delusions. W. M. MeLaury. Alienist and Neurol., St. Louis, 1894, xv, 429-454.

Das Delirium halluciuatorinm. E. Mendel. Berl. klin. Wchnschr., 1894, xxxi, 663-667.

- Ueber nnilaterale Hallucinationen. H. Higier. Wien. Klinik, 1894, $x x, 189-170$.

- Lidt om Hallucinationer. H. Holm. Norsk. Mag. f. Laegeirclensk., Christiania, 1894, 4 R., ix, 433-454.

- Considérations sur les hallucinations provoquées par la perte d'un sens, a propos d'un cas de délire complexe avec hallucinations multiples consécutif à la perte de la rue chez un dément sénile. France méd., Par., 1894, xli, 869, 386.

Sur un cas d'hallucinations motrices verbales chez une puralytique générale. P. Sérienx. Arch. de neurol., Par., 1894, xxvii, 321-352.

Les hallucinations et le de doublement de la personnalité dans la folie asstématique; les persécutés possédés et la variété psycho-motrice du délire des persécutions systématique. Ann. méd.-peych., Par., 1894, 7 s., $x \times, 5-43$.

- Ueber die Trugwuhrnehmung (Hallucination und lllnsion) mit besonderer Berücksichtigang der internationulen Enquête über Wachlallucinationen bei Gesunden. Schrift. d. Gesellsch. f. p\&ychol. Forschr. E. Parish, Leipz., 1898-4, ii, 49.228, 31.

Headache. De la céphalalgie dans les maladies mentales. A. Cullerre. Aun. med.-paych., Par., 1894, 7 s., xx, 75-83.-Par., 1894, xxviii, 2-11.

Heart. Die Hypoplasie des Herzens bei Geistesschwachen. Wulff. Allg. Ztschr. f. Paychiat., \&c., Berl., 1894, li, 447-457.

Heart lesions in mental diseases. H. D. Vulin. Chicago Clin. Rev., 1893-4, iii, 828-348.

- Oeber die nnatomischen Veränderungen des Herzens bei chronischen Geistesstorrungen; eine statische Untersuchung. C. Strecker. Arch. f. path. Anat., \&c., Berl., 1894, cxxxvi, 217-244.

- Ueber organisclie und functionelle Herzleiden bei Geisteskranken. Mün. chen. med. Wchnschr., 1894, xli, 305, 328, 355. G. Reinhold.

Histology. Sur les altérations histologiques de l'écorce cérébrale dans quelques maladies mentales. R. Colella. Arch. ital. de biol., Turin, 1893-4, xx, 216 218.

Hyoscine. Hyoscinæ hydrobromas in a case of old-standing mania; extraordinary result of a single large dose. Balagopal. Indian M. Rec., Calcutta, 1894, vi, 143.

Hysteria. Hysteria. A. Mstislavski. Feldscher, St. Petersb., 1894, iv, 142. 146.

On a case of hysterical alexia, cured by suggestion. B. A. Pope. N. Orl. M. and S. J., 1893-4, n. \&., xxi, 814.816.

Antomatiome ambulatoire de nature hystérique ; présentation de malade. Régis. Mém. et bull. Soc. de méd. et chir de Bordesux (1893), 1894, 31 43.

- Afonia isterica. F. Ruedu. ['Transl.] Boll. d. mul. d. orecchio, d. gola ed. ияво, Firenze, 1894, xii, 82-85.

Febbre istericu come equivalente termicu dell' accesso. F. Santangelo Spoto. Gazz. d. osp., Milano, 1894, xv, 177-181.

Etiologie infectieuse de l'hystérie. Grasset. N. Montpel. méd., 1894, iii, 409, 431.

Ueber einen schweren Fall von Hysterie. J. Hackel. St. Petersb. med. Wchnschr., 1894, n. F., xi, 163-165; 1 pl.

- Beitrag zur hrsterischen Apoplexie. H. Higier. Wien. klin. Wchnschr., 1894, vii, 823, 847, 888.

Ueber hysterische Muskelatrophie. L. Hirt. Dentsche med. Wchnschr., Leipz. u. Berl., 1894, xx, 459. 
Hysteria. Hysterie und ihr Einfluss auf die Malerei. F. Horn. Wien. klin. Wehnschr., 1894, vii, 295, 314.

Studio sopra un cuso di spasmo della glottide da isterismo. S. Lumbau. Gazz. med. lomb., Milano, 1894, liii, 131, 141, 153.

Sur trois cas d'hystérie infantile. Bernheim. Rev. méd. de l'est., Nancy, 1894, xxvi, 313-316.

- Gangraens cutis hysterica. F. Ehrl. Wien. klin. Wchnschr., 1894, vii, 330-333.

Simulation hystérique. J. Verhoogen. J. de méd., chir., et pharmacol., Brux., 1894, 38.

- Contribution à l'étude des pseudoméuingites hystériques (symptomatologie et diagnostic). Brugère. Bordesux, 1893, 57 pp. 4to, N1. 47*.

Etat mental des hystériques. Les accidents meutaux. Pierre Janet. Par., 1894, Rueff' et Cie., 304 pp. $12 \mathrm{mo.}$

Hysterie und Suggestion. Munchen. med. Wchnschr., 1894, xli, 117, 146. L. Löwenfeld.

- Su due casi d'isteria maschile nssocinta u pazzia morale. S. de Sanctis Policliv, Rowa, 1893-4, i, 266-273.

Nute sur la gangrène spontanée de la peau chez les hystériques. C. Féré. Compt.-rend. Soc. de Biol., Pur., 1894, 10 s., i, 427-429.

Mutismo isterico gunrito con l'eterizznzione. Progresso uned. C. Gioffredi. Napoli, 1894, viii, 119-130.

Die Behandlung der Hysterie. E. Hecker. Therap. Monatsh., Berl., 1894, viii, 143, 206.

['Two cases of hysteria, simulating Purkinson's and Thomson's disease.]

G. A. Kliatchkin. Med. Obozr., Mosk., 1894, xli, 275-282.

Does nystagmus exist in hysteria? J. Sabrazès. Med. Week, Par., 1894, ii, 409.

Un caso de histeri: con temperatura de $105 \cdot 8^{\circ}$ F. J. Stufford, Rev. méd. quir. amer., N.Y., 18y4-5, iii, 2.

- Some notes on hysteria, with special reference to hysteria in the mule, and its connection with specific organic disease of the nervous system. A.S. Walker. Edinb. M. J., 1894-5, xl, 312-322.

- Beiträge zur Casuistik der Hysteria virilis. Carl Gergen. Wurzburg, 1893, J. Seelmeyr, 56 pp. 8vo.

[Hysteria in soldiers.] Vrach, St. Petersb., 1894, xv, 597, 624. M. B. Blumentu.

Hysteria and Neurasthenia. J. M. Clarke. Brain, Lond., 1894, xvii, $119-178$

- Vomissement incoërcibles depuis 10 mois, chez une jeune fille de 14 ans ; hystérie; guérison rapide des vomissements après suggestion bypnotique. Dumontpallier. Rev. de l'hypnot. et psychol. physiol., Par., 1894-5, ix, 21.

- Surdi-ınutité hystérique guérie par suggestion à l'état de veille. $X$. Francotte. Ann. Soc. méd.-cliir. de Liége, 1894, xxxiii, 285-289.

[A curious case of hysteria in man]. 'T. Hondo mada I. Kokuwa. Chugai Ijishimpo, Tokio, 1894, No. 331, 6 ; No. 332, 15; No. 333, 14.

Un cas de mutisme h!stérique. Marotte. Prorince méd., Lyon, 1894, viii, 289-291.

Hysteria and neurasthenia. A. B. Richardson. Columbus M. J., 1894-5, xii, $131-137$.

- Contribution à l'étude de l'hystérie toxique (intoxication sulfo-carbonée). Charles Martel. Par., 1894, G. Steinheil. 60 pp. 8vo.

- Des rapports de l'hystérie et de la folie. Ballet. [Rsp.] Limousin méd., Limoges, 1894, xviii, 133-136.

The relation of hysteria to structural changes in the nterus and its adnexa. A.P. Clarke. Ann. Gynæc. and Pædiat., Phila., 1894-5, viii, 55-57. [Hysteria in the Army; new case of, in a soldier]. B. S. Greidenberg. Vrach, St. Petersb., 1894, xv, 736-738.

Ueber Hysterie im Kindesalter. A. Hoffmann. Fünfz. Beitr. a. d. Geb. d. ges. Med. Festschr. . . . d. Ver. d. Aerzte d. Rgrngsbz. Düsseld., Wiesb., 1894, 182-189. 
Hysteria. Isterrimo, lezioni cliniche. Samuele Gubitosi. Napolie, 1893, Valeris and Silvestri, 178 pp. 8vo.

- A case of hysteria in a boy, characterised by regularly recurring attacks of lethargy ; treatment by hynotism. T. Diller. Brain, Lond., 1893, xvi, 556.561.

Hysterical aphasia, hemianæsthesia, and dyschromatopsia. C. K. Mills. Phila. Hoep. Rep., 1898, ii, 148-151.

- Psendo-globus hystericus. P. J. Mink. Geneesk. Courant, Tiel 1893, Ixvii, No. 48.

Neurosis in women; (hysteria). F. B. Robinson. Gaillard's M. J., N. Y., 1894, lviii, 103-108.

Hysteria. L. G. Smart. N. Albany M. Herald, 1893, xiii, 748-752.

- Observations de mensonges ou prétendus mensonges des hystériques.

C. Vibert. Ann. d' hyg., Par., 1894, 3 s., xxxi, 171-181.

A case of male hysteria characterised by recurrent attacks of motor aphasia and lethargy; apparent cure by hypnotism and suggestion. T. Diller. Internat. M. Mag., Phila., 1894-5, iii, 182-186.

Four weeks starvation caused by lysteria; cured by a sight at the stomach pump. (Lincoln's Inn.) Jugganath. Indian M. Rec., Calcutta, 1894, vi, 176.

A case of hysteria. A. L. Knight. Cincin. Lancet-Clinic, 1894, n. 8., xxxii, 513, [Discussion] 522.

- Quatro casos de hysterir em homens. M. Nery. Brazil-med., Rio de Jan., 1894, 65.67.

- Un cas de pseudo-6léphantiasis névropathique du membre supérieur chez une femme hystérique. $G$. Thibierge. Bull. et mém. Soc. méd. d. hốp. de Par., 1894, 3 s., xi ; 262-268.

Observations de mensonges ou prétendus meusonges des hystériques. C. Vibert. Rev. de méd. lég., Par., 1893.4, i, 241.246.

Beitrag zur Casuistik der Hysterie. A. Bäumel. Wien. med. Presse, 1894, $\times x \times v, 1010-1014$

L'état mental dans l'hystérie. P. Blocq. Gaz. d. hôp., Par., 1893, lxvi, 1278-1280.

- Des tremblements hystériques. P. Delmar. J. de Méd. de Bordeaux, 1898, xxiii, 437, 470, 483, 491, 503, 517.

Associazione dell' isterismo coro lesioni organiche dell' arecchio. G. Gradenigo. Gazz. d. osp., Milano, 1893, xiv, 1218.

La théorie psychologique de l'hystérie (formale cortico cérébrale de cette névrose), revue générale. N. Grasset. Montpel. méd., 1893, iii, 866, 885.

Hyeterical blue odema. A. J. McCosh. Ann. Surg., Phila., 1893, xviii. 667.

Maskerede Hysterier oj hysteriske Masker. K. Pontoppidan. Hosp. Lid. Kjobenh., 1893, 4 R., i, 741.750.

Da tremblement hystérique. Roger. Semaine méd., Par., 1893, xiii, 522. Also Trans. Med. Week, Par., 1893, i, 537.

Hysterical trembling, stammering, and mutism. J. C. Shaw. Internat. Clin., Phila., 1893, 3 s., iii, 118.123.

Storia clinica di au isterica, conpilegia traumaticoisterica; nevrosi cardiaca ; accessi catalettici; grandi convulsioni; afonia ; grave spasmo accessionale della glottide; considerazioni sulla cura fatta colla pilocarpina e colla suggestian. G. Spreza. Raccogliton med., Fœli, 1893, 5 S., xvi, 289-302.

Hysterical disturbances of the special senses. J. H. Thompson. Tr. M. Ass. Missouri, Jefferson City, 1893, 166-172.

Un caso de histerismo. R. del Valle q Aldabalde. Rev, de med. q cirug. pract., Madrid, 1893, xxxiii, 281-286.

Isterismo e sentimento. Giuseppe Cattani. Milano, 1894: L. Omodei Zorini, 369 pp. 12 mo.

Un caso de histerismo en un joven de diecinueve años. P. Peñarredonda y Barrio. Siglo méd., Madrid, 1894, xli, 517.

- L'hyst6rie paludéenne. H. Bidon. Marseille méd., 1894, xxxi, 513-536.

Vomissements, incoercibles chez une hystérique de 14 ans, guérison par suggestion. Dumontpallier. Courrier méd., Par., 1894, xliv, 244. 
Hyateria. Note sur l'oligodactylie cubitale chez les hystériques. C. Féré et J. Roger. Compt. rend. Snc. de Biol., Par., 1894, 10 s., i, 619-621.

- Toux nerveuse; coxalgie hystérique; unorexie; contractures multiples troubles pxychiques chez une jeune tille. Buzaucaes de Guesdron. J. de clin. et de thérup. inf., Par., 1894, ii, 752-754.

Case of luysteris in a child, with remarkable psjchical symptoms. H. G. Lungwill. Edin. Hosp. Rep., 1894, ii, 4:26-429.

Sur un cas d'hystérie à forme particulière. R. Lépine. Rev. de méd., Pur., 1894, xiv, 713.728.

Un cas de mutisme hystérique. M. Natier. Ann. de la Policlin. de Par., 1894, iv, 256-262.

- Disharmony between pulsation and impulse of the henrt in inorbid stuges of hysteria. F. Sorustein. Medycynн, Wnrsznwa, 1893, хxi, 962-964.

L'hystérie a Toulon, vers 1730. H. Bidow. Marseille méd., 1893, xxx, 521, 537, 567, 577, 616 .

- Some notes on hysteria, with special reference to hysterin in the male and its connection with specitic organic disense of the uervous system. A.S. Walker. Edinb. M. J., 1894-5, x1, 312-322.

The gravity of hysteria. G. Eliot. N. York M. J., 1894, Ix, 390-392.

- Ueber einige Symptome der Hysterie und über die Beziehungen der Hysterie zum Alkoholismus. Ganser. Juhresb. d. Gesellsch. f. Nat. u. Heilk. in Dresd., 1893-4, 119-133, [Discussion] 7y-83.

- L'hystérie dans l'urt antique; étude inédicale sur quelques monuments figurés de l'antiquité. H. Meige. Internat. med. phot. Monatschr., Leipz.; $1894, \mathrm{i}, 187,167$.

Zur Patholngie des hysterischen Stotterns. E. Remak. Berl. klin. Wehnschr., 1894, x×xi, 795-797.

Grunde isteria con crisi convulsive ; contratture, sordità, cecità ; dolori all' ovaia; guarigione coll' ipnotismo. E. Terrien. Ipnotismo, Firenze, $1894, v, 163$.

- Des éruptions cutanées chez les hystériques. Adolphe Gauthier. Lyon, 1893,69 pp. 4to, No. 801 .

Hysterical conditions, with clinical history of a case. A. Halliday, Mnritime M. News, Halifax, 1894, vi, 211-214.

- A further study of hysterical cases and their fields of vision. J. K. Mitchell. J. Nerv. and Ment. Dis., N.Y., 1894, xxi, 1-49.

- Des fansses paraplégies chez lea garcons hystériques. J. Simon. Bull. méd., Pur., 1893, vii, 1131-1133; also Progrès méd., Pur., 1894, 2 8., xix, 1.4.

De l'hystérie en Vendée. Terrien. Arch. de neurol., Par., 1893, xxvi, $447-475$

- Strange hysterical fits in a young man. J. Benjamin. Indian M. Rec., Calcutta, 1894, vi, 144.

Hypnotismus und Hysterie: eine Erwiderung. München. med.

Wchischr. F. Jolly. 1894, xli, 247.

Spasmo clonico dei retti addominnli $d^{\prime}$ indole isterica. T. Legnani. Arch. Ital. di clin. med., Milano, 1893, xxxii, 566.

- Hysterie mit Schmerz- und Krampfanfallen bei einem 12 jahrigen Mädchen. Heilung-Mader. Wien. med. Bl., 1894, xvii, 101.

Ein Fall von hysterischen Mutismus. G. Ringier. Ztschr. f. Hypnot., \&c., Berl., 1893-4, ii, 143-150.

Hystero-epilepey. Report of a case of hystero-epilepey cured by operative interference. L. A. Malone. Tri-State M.J., Keokuk, 1893-4, i, 201-204. A case of hystero-epilepey in a man. J. B. Thornton. Boston M. and S. J., 1894, cxxxi, 416.

- Di una rara forma di impulaione-ambulatoria in istero-epilettico per traumatismo. G. Antonini. Riv. sper. di freniat., Reggio-Emilia, 1894, xx, pt. 2, 198.203.

Die Hysteria nach den Lehren der Snlpêtrière. Mit einem Vorwort von J. M. Charcot. Autoriserte deutsche Aurgabe von Dr. Karl Grube. Normale oder interparoxysmale Hysterie. Gilles de la Tourette. Leipz. und Wien, 1894, F. Deuticke, 842 pp., 8 vo. 
Hysteria. Der Geisteszustand der Hysterischen (Dic psychischen Stigmata). Mit eines Vorrede von Profeseor Charcot. Uehersetzt von Dr. Mnx Kuhane. Pierre Janet. Leipz. und Wien, 1894, F. Deuticke, 197 pp. 8vo.

- $\Delta$ hyoscin hatása a hysteroepilepsiás roham ellen. [Action of hyoscin in hyotero-epileptic attack.] Orvo-i hetil., Budapest, 1893, xxxvii, 484, 498, 613, 624. Also, transl. [Abstr.]. Pest. med.-chir. Presse, Budaprs', 1894, $x \times x, 172 ; 197$. N. Béla.

Report of a cuse of hrstrro-epilepsy. I. J. Bush. Texas Cour.-Rec. Med., Dällas, 1893-4, xi, 191.193.

Sluch. reflektor. hystero-epilepsiæ. S. N. Koneff. Med. besiedn, Voı onej, 1894, viii, 821.826.

Isteru-epilessia da Influenza. N. Mnsmeci. Riv. clin. e terap., Napoli, 1894, xvi, 402-40R.

Idiocy. Zur Aetiologie der Idiotie. Mit einem Vorwort von Dr. W. Sander. Hermann Piper. Berl., 1893, Fischer, 207 pp. 8vo.

Du traitement chirurgical et du traitement médico-pédagogique de l'idiotie. Bourneville. Cong. ann. de méd. ment., C.-r., 1892, Blois, 1893, iii, 261.303.

Types of idiocy and imbecility. F. Beach. Clin. J., Lond., 1894, iv, 48.46.

L'idiotie et l'imbécillité au point de vue nosographique. P. Sollier. Arch. de neurol., Par., 1894, xxvii, 33-38.

Le forme dell' idiotismo. E. Morselli. Anomalo, Napoli, 1893, v, 5-11. Limitations in the treatment of the idiot. B. Jones. Am. J. Surg. and Gynec., Kansas City, 1893.4, iv, 240.243.

(A) Discussion on points connected with the education of feeble-minded children. Brit. M. J., Lond., 1894, ii, 528-581.

Conformation des organes génitaux chez les idiots et les imbéciles. J. Voivill. Ann. d'hyg., Par., 1894, 3 s., xxxi, 525-532. Périodes régulières d'excitation maniaque et de dépression mélancolique chez une idiote de huit ans. Bull. méd., Par., 1894, viii, 505.507.

Cas d'idiotie symptomutique d'une atrophie de l'hémisphère gauche (lobes parietaux ct occipituux). Homery. Ann. méd.-psych., Par., 1893, 7 a.. xriii, 428-431.

Considérations sommaires sur le truitement médico-pédagogique de l'idiotie. Bourneville. Méd. inf., Par., 1894, i, 16-38.

Two interesting cases of idiocy. 1. Idiocy with spastic and athetoid phenomena. 2. Rachitic idiocy. W. Sinkler. Phila. Hosp. Rep., 1893, ii, 161-168.

[Syphilitic spinal paralysis in connection with imbecility.] A. Popoff. Ruenk. Med., St. Petersb., 1893, xviii, 735, 755, 775.

Some clinical aspects of imbecility. S. J. Fort. Maryland M. J., Balt., 1894, xxxi, 123-127.

Imperative ideas. D. H. Tuke. Brain, Lond., 1894, xvii, 179-197.

Zur Theorie der Zwangsvorstellungen. Allg. Ztschr. f. Psychiat., \&c., Berl., 1898-4, 1, 1063-1074.

Infectious processes. The relations of infectious processes to. mental disease. C. K. Mills. Am. J. M. Sc., Phila., 1894, n. 8., criii, 563.517.

Insanity. Ueber das inducirte Irresein (Folie communiqu6́). M. Schønfeldt. Arch. f. Peychiat., Berl., 1894, xxvi, 202-266. - La folie post-opératoire. Jacobs. Presse méd. belg., Brux., 1894, xlvi, 233.

- Prophecy and insanity. J. Weir, jun. Am. Pract. and News, Louisville, 1894, xviii, 89-106.

The increase of insanity in Virginia, and practical suggestions for the care and maintenance of insane people by the State. J. 8. Apperson. Virginis M. Month, Richmond, 1894-5, xxi, 801-318.

The hiotory of an experiment in dealing with the reported cases of insanity occurring in the Barony Parish of Glasgow. J. Carewell. J. Ment. Sc., Lond., 1894, xl, 394-404. 
Insanity. Diagnostik der Geisteskrankheiten für praktische Aerzte und Studirende, Wien und Leipz., 1894, Urban nnd Schwarzenberg. Robert Somner. 310 pp. 8vo.

- Folie consécutive a une ovarosalpingectomie double; présentation de malade. Mém. et bull. Soc. de méd. et chir. de Bordeaux (1893), 1894, 279-291. Régis.

- Sul peso specifico dell' orina nelle malattie mentali. U. Stefani. Riv. sper. di freniat., Reggi-Emilia, 1894, xx, 1-7, $1 \mathrm{ch}$.

Problems of public interest concerning the insane. Opheua Everts. Read before the Missisoippi Valley Medical Association, Oct. 4, 1893. Cincin., 1893, R. Clarke and Co., 11 pp. 8vo.

-__ Excessive mental excitement, und some of its consequences. T. R. Glynn. Liverpool M. Chir. J., 1894, xiv, 1-21.

[The crimes of the insane from excessive nervous irritation.] V. N. Ergolski. Arch. psichiat., \&c., Charkov, 1894, xxiii, 60-68.

De l'hypothermie chez les aliénés. J. B. Bouchaud. Ann. méd.-psych., Par.. 1894, 7 s., xix, 194-265, 4 ch.

- Sulle cause della odiema anarchir psichica e compito dell' igiene morale

di comisatterle. Landerer. Riv. internaz. d'ig., Napoli, 1894, v, 145-160.

I tremori nei pazzi. A. Cristiani. Riv. sper. di freniat., Reguio-Emilia, 1894, xx, 17-22, $1 \mathrm{ch}$.

On the means of preventing and evading insanity. W. W. Ireland. Alienist and Neurol., St. Louis, 1894, xv, 181-208.

Les folies des maladies aigües. et Chevalier-Lavaure Regis. Gaz. hebd. de mód., Par., 1893, xl, 457, 471, 484.

- De la folie par commotion cérébrale et de ses rupports avec la législation militaire. A. Rigal. Ann. d'hyg., Par., 1894, 3 s., xxxi, 204, 340.

Report of fifty-seven cases of insanity occurring in six years among silk-mill employées in a manufacturing city in New Jersey. W. P. Spratling. N. York M. J., 1894, lix, 614-616.

Ueber Beziehung moderner Zeitströmungen zum Irrsinn. Blıustern. Deutsche Rev., Stuttg., 1894, 256-258.

Ueber die Formen von Geistesstörung, welche Hexenprocesse veranlasst

haben. O. Snell. Allg. Ztschr. f. Psychiat., \&c.. Berl., 1893-4, 1, 534-545.

Ueber inducirtes Irresein und über einzelne inducirte und ahnliche Erscheinungen bei Geisteskranken. Roller. Allg. Ztschr. f. Psychiat., \&c., 1893-4, 1, 722-739.

The institutional care of the insane. E. P. Christian. Physician and Surg., Detroit and Ann Arbor, 1894, xvi, 208-215.

Morison lectures on insanity. J. B. Tuke. Edinb. M. J., 1893-4, xxxix, $673,769,890,983,1090,1103,1 \mathrm{pl}$.

The physical hasis of insanity and the insane diathesis. W. A. White. Am. J. Insan., Utica, N. Y., 1893-4, 1, 530-537.

- Ueber die Gewebsvereänderungen der Nervenzellen in der Hirnrinde bei Geisteskrankheiten. B. Nagy. Ungar. Arch. f. Med., Wiesb., 1894, iii, 17-37, 1 pl.

Calvarium from a case of circular insanity. L. R. Oswald. Glasgow M. J., 1894, xlii, 61-63.

- On three cases of recovery after a lengthened duration of insanity, with remarks. J. A. Campbell. J. Insan., Utica, N. Y., 1893-4, 1, 505-511.

- 'The commitment, detention, care, and treatment of the insane in America. G. A. Blumer. Am. J. Insan., Utica, N. Y., 1893-4, 1, 538-556.

The insane kings of the Bible. D. R. Burrell. Am. J. Insan., Utica, N. Y., 1893-4, l, 493-504.

Le problème de l'assistance des aliénés. E. Toulouse. Gaz. d. Lıôp., Par., 1894, Irvii, 177, 199.

On the early stages of acute insanity. T. C. Shaw. St. Barth. Hosp. Rep., Lond., 1893, xxix, 109-123.

- The inndequacy of the morbid changes found post mortem to explain the manifestations of insanity. H. A. Tomlinson. Univ. M. Mag., Phila., 1893-4, vi, 657.672. 


\section{JOURNAL OF MENTAL SC, NCE. CONTENTS FOR JANUARY, 1899.}

Original Articles.-

1. A. R. Douglas, - The Improvable Imbecile, his Tr

2. David Bower, M.D. - The Inadequate Asylum Provision for the Insane immediately above the Pauper Class

3. J. G. Havelock, M.D. - The Workmen's Compensation Act and the Fatal Accidents Inquiry (Scotland) Act in Relation to Asylums

4. J. G. Gordon-Munn, M.D., F.R.S.E.-A Communication on the Appearances of the Uterus and its Appendages in the Insane.

5. Jas. Middlemass, M.D., F.R.C.P.E.-On the Treatment of Insanity by Thyroid

6. Reginald H. Noott, M.B., C.M.-The Responsibility of the Insane; should they be punished? A Reply to Dr. Mercier

7. H. C. Bristowe, M.D.-Lunacy in Private Practice

8. Edwin Stephen Pasmore, M.D.Lond., M.R.C.P.Lond.-Observations on the Classification of Insanity .

9. Moriz Benedikt.-The Waldstein Case at Prague

I0. W. W. Ireland, M.D.-Further Research on the Formation of Axial Fibres

in the Brain, by Dr. Paul Flechsig, Dr. Döllken, and Dr. Nissl. A Digest 87

Clinical Notes and Cases.-

A Case of Juvenile General Paralysis; by A. Helen Boyle, Ir.D. - The

Effects of an Injury to the Head in an Alcoholic Subject; by N. Raw, M.D.

-Pathological Notes on Cases of Heart Disease at the Durham County

Asylum; by T. Aldous Clinch, M.D.

Occasional Notes.-

The Lord Chancellor's Lunacy Bill,-Criminal Evidence Act.-The Case of

Dr. J. A. Campbell.-Luccheni : the Murderer of the Empress of Austria -

The Bedborough Case.-Pathological Laboratories.-The Innervation of Intra-cranial Blood-vessels.-Phthisis in Asylums and the Segregation of Phthisical Cases.-The Psychological Section at the Edinburgh Meeting.Drunken Women.-The Frederick Case.-Over-burdened Children in Germany.-Juvenile Criminals.-Punishment of the Insane,-Lunacy in New Zealand . $114-13^{8}$

\section{Reviews.-}

Report of the Commissioners in Lunacy for England,-Report of the Commissioners in Lunacy for Scotland.-Report of the Inspectors of Lunatics for Ireland. - The Origin and Growth of the Moral Instinct; by Atexander Sutheri.and, M.A.-Mental Affections of Children; by W. W. Ireland, M.D.-Recherches cliniques sur l'Épilepsie, l'Hystérie, et 1'Idiotie; par Bournevit.te.-Les Maladies de 1'Esprit; par Dr. P. MAx-Srmon,-Le Róle du Patronage à l'égard des Aliénés; par Dr. Jules Morel.-L'Année psychologique.-Forensic Medicine and Toxicology; by J. Dixon Mann, M.D.-Guide to the Clinical Examination and Treatment of Sick Children: by Jorn Thomson, M.D.-On a Method of collecting the Pathological Statistics of the Insane Brain; by FrANcis O. Stmpsos, L.R.C.P.-Hysteria as a Psychosis; by Dr. Donald Fraser

Psychological Retrospect.-

French Retrospect; by Dr. Macevoy . . . . . . . . . . . . . .

Notes and News.-

Medico-Psychological Association of Great Britain and Ireland: General Meeting; South-Eastern Division; South-Western Division; Northern and Midland Division; Scottish Division.-British Medical Association: Edinburgh Meeting.-Quebec Medico-Psychological Society.-Recent Medico-Legal Cases. - After-care Association. - The Journal. - Craig Colony Prize for Original Research in Epilepsy.-Resignation.-Presenta. tions. - The Welfare of the Feeble-minded. - A "Colony" for Epileptics and Imbeciles in Lancashire.-Sewage Disposal. - The Treatment of Imbeciles and Epileptics.-Should Imbeciles Work?-Mental Suffering.-L'intermédiare des Neurologistes et des Aliénistes. - Correspondence. - The Lunacy Commission.-Obituary. - Notices by the Registrar.- The Prize Dissertation.

-Notices of Meetings.-Appointments . . . . . $189-224$

ADLARD AND SON, PRINTERS, BARTHOLOMEW CLOSE, E.C., AND 2O, HANOVER SQUARE, W. 